\title{
NEGATIVE URINE PREGNANCY TESTS IN GESTATIONAL TROPHOBLASTIC DISEASE: A DIAGNOSTIC DILEMMA
}

Jayanta Ray로 Ashis Kumar Rakshit², Soumitra Majumder³, Salil Bindu Chakrabarty ${ }^{4}$

\section{HOW TO CITE THIS ARTICLE:}

Jayanta Ray, Ashis Kumar Rakshit, Soumitra Majumder, Salil Bindu Chakrabarty. "Negative Urine Pregnancy Tests in Gestational Trophoblastic Disease: A Diagnostic Dilemma". Journal of Evolution of Medical and Dental Sciences 2014; Vol. 3, Issue 35, August 14; Page: 9241-9242, DOI: 10.14260/jemds/2014/3192

ABSTRACT: Gestational trophoblastic disease is a broad disorder of abnormal fertilization of ovum. Early detection and treatment is the best approach to prevent mortality and morbidity of patients of gestational trophoblastic tumors. All trophoblastic cells produce hcg (human chorionic gonoadotropin) and can easily be detected in urine of pregnant ladies. When urine pregnancy test become negative in a highly suspected patient, the management becomes difficult. In this case, though the urine pregnancy tests were negative on several occasions, serum $\beta$ hcg level was very high $>1500000 \mathrm{mIU} / \mathrm{ml}$ (positive) and patient was cured by chemotherapy.

KEYWORDS: Gestational Trophoblastic Tumor, Urine Pregnancy Test, $\beta$ hcg, chemotherapy.

INTRODUCTION: Gestational Trophoblastic Disease (GTD) is a broad term for group of disorders of pregnancy complications arising from abnormal placental trophoblast cells. Commonly GTD presents with bleeding, excessive pregnancy symptoms like hyperemesis gravidarum. Some patients do present with uterine size more than that of period of gestation. All such presentations are suspected and diagnosis is confirmed by good history and investigations like urine pregnancy test and Ultrasonography of lower abdomen. In some cases the presentation of these diseases may not be typical and makes the diagnosis and management difficult for the clinician. The case reported here is one such condition.

CASE REPORT: Twenty year old tribal woman from a poor socio economic background reported to labor room with complaints of pain and swelling in lower abdomen, off and on bleeding per vagina since Medical Termination of Pregnancy three months back and thereafter, had repeated Dilatation \& Curettage (D \& C) for incomplete abortions. Earlier One year back she gave birth of a live born girl in a rural hospital. The couple did not adopt any contraceptive methods after the child birth.

The patient was admitted in labor room with a provisional diagnosis of twisted ovarian tumor and incomplete abortion. Her urine pregnancy tests on four occasions were negative. Ultrasonography suspected molar pregnancy with hyper stimulated both ovaries. Serum $\beta$ human chorionic gonadotropin ( $\beta$ hcg) level was $>1500000 \mathrm{mIU} / \mathrm{ml}$. So the patient was leveled as a case of Gestational Trophoblastic Tumor (GTT). The patient was given chemotherapy EMACO regimen. After the full chemotherapy, she got cured and later discharged from the hospital. She is now healthy and under follow up.

DISCUSSION: Gestational Trophoblastic Disease is a broad diagnosis for different types of pregnancy related disorders arising from abnormal placental trophoblastic cells. GTT can be either benign or malignant. All forms of trophoblastic cells produce $\beta \mathrm{hcg}$. Amount of $\beta \mathrm{hcg}$ is directly proportional to the amount of disease load. There is geographical variation of the GTD. Incidence in Asia is higher 
than in western countries. ${ }^{1}$ The disease is more common in younger women and women nearing their end of reproductive period. ${ }^{2}$ Current urine and serum pregnancy tests use antibodies directed against $\beta$ hcg for immunologic identifications.

Two antibodies directed against different parts of $\beta$ chain are used, and resultant "sandwich" they form around the antigen is detected and interpreted as 'positive'. The most test kits have one antibody to site $\beta 1$ and another to the $C$ - terminus of the $\beta$ - subunit ( $\beta$ - CTP), or to $\alpha$ subunit.

However, falsely low or negative result can occur when an extremely high level of $\beta$ subunit core fragment, a breakdown product of $\beta$ hcg overwhelms the assay system and blocks the formation of "sandwiches" between the two sites. ${ }^{2,3}$ This is also called "hook effect" or "prozone phenomenon" can be observed in immunometric sandwich assay. This hook effect can be overcome simply by dilution method if suspicion is high. ${ }^{4}$

\section{REFERENCES:}

1. Bracken MB. Incidence and aetiology of hydatidiform mole: an epidemiological review. $\mathrm{Br} \mathrm{J}$ Obstet Gynaecol 1987; 94: 1123-35.

2. Er Tk, Tsai LY, Gines Ruiz MA. Quantitative human chorionic gonadotropin measurement in urine using the Access immunoassay. Am J Emerg Med 2008; 26: 103-4.

3. Yunus D, Muppala H, Hamer F, Clarke F. Three consecutive false negative pregnancy tests in a twin pregnancy: a case report. Int J Gynecol Obstet [serial online] 2007; 6. Available at: www.ispub.com/journal/the_journal_of_gynecology_and_obstetetrics/vol_6_number_2_6/articl e/three_cosecutive_false_negative_pregnancy_tests_in_a_twin_Pregnancy_a_case_report.html.Ac cessed February 102010.

4. Wolf BA, Garrett NC, Nahm NH. The "hook effect": high concentration of prostate- specific antigen giving artifactually low value on one- step immunoassay. N Engl J Med 1989; 320: 1755-6.

\section{AUTHORS:}

1. Jayanta Ray

2. Ashis Kumar Rakshit

3. Soumitra Majumder

4. Salil Bindu Chakrabarty

\section{PARTICULARS OF CONTRIBUTORS:}

1. Associate Professor, Department of Obstetrics and Gynaecology, AGMC.

2. Assistant Professor, Department of Obstetrics and Gynaecology, AGMC.

3. Assistant Professor, Department of Obstetrics and Gynaecology, AGMC.

4. Assistant Professor, Department of Obstetrics and Gynaecology, AGMC.

\author{
NAME ADDRESS EMAIL ID OF THE \\ CORRESPONDING AUTHOR: \\ Dr. Jayanta Ray, \\ Palace Compound, \\ Thakur Anil Krishna Sarani, \\ Tripura-799001. \\ Email: drjayantray@gmail.com
}

Date of Submission: 26/07/2014.

Date of Peer Review: 27/07/2014.

Date of Acceptance: 06/08/2014.

Date of Publishing: 13/08/2014. 